\title{
Liver Transplant for Patients with Hepatocellular Carcinoma and Child-Pugh C Liver Function Should Be Mentioned in Guidelines
}

\author{
Xu-Feng Zhang ${ }^{a, b} \quad$ Yi Lva, b \\ aDepartment of Hepatobiliary Surgery and Institute of Advanced Surgical Technology and \\ Engineering, The First Affiliated Hospital of Xi'an Jiaotong University, Xi'an, China; ${ }^{\text {b National }}$ \\ Local Joint Engineering Research Center for Precision Surgery and Regenerative Medicine, \\ The First Affiliated Hospital of Xi'an Jiaotong University, Xi'an, China
}

Dear Editor,

We read with great interest the latest publication "Guidelines for Diagnosis and Treatment of Primary Liver Cancer in China (2017 Edition)" in Liver Cancer [1]. The guidelines have first been published in 2011 and were then updated periodically $[1,2]$. The guidelines have been discussed and drafted by the most famous physicians and scientists in the field of liver cancer prevention and treatment in China. The guidelines have mostly been adopted in the diagnosis and treatment of hepatocellular carcinoma (HCC) by Chinese physicians and scientists.

In the guidelines, screening, diagnosis, staging, and series of treatments have been thoroughly discussed, and standardized protocols have been recommended. For example, a detailed guideline for HCC treatment has been proposed in Figure 2 of the article by Zhou et al. [1]. However, for patients with HCC and poor liver function (ChildPugh C status), only symptomatic supportive treatment and palliative treatment/care have been recommended. As known, the absolute number of HCC cases and HCC-related death accounted for half of the HCC cases all over the world [3]. In contrast to HCC in developed countries, $60-70 \%$ of the HCC in China developed due to hepatitis $B$ virus infection and subsequent liver cirrhosis [4]. As such, the resection rate of HCC was only 30-40\% [4]. Many patients with HCC presented with a high tumor load and/or decompensated liver cirrhosis, making curative resection difficult. In fact, in patients with Child-Pugh Cliver function, if the tumor load meets certain criteria, such as the Milan criteria, University of California San Francisco (UCSF) criteria, Fudan criteria, and/or Hangzhou criteria, liver transplant should be considered the last possible measure and the optimal treatment for this group of patients [3]. Although liver transplant (UCSF criteria) has been added as a treatment for HCC of stages Ia, Ib, and IIa, it has not been mentioned as a curative treatment for HCC in patients with Child-Pugh C liver function.

We appreciate any comments from and discussion with liver cancer experts, physicians, and scientists and appreciate their great contribution to the progression in the control, diagnosis, and treatment of HCC in our country and all over the world.

\section{Disclosure Statement}

The authors have no conflicts of interest to declare. 


\section{References}

1 Zhou J, Sun HC, Wang Z, Cong WM, Wang JH, Zeng MS, et al. Guidelines for Diagnosis and Treatment of Primary Liver Cancer in China (2017 Edition). Liver Cancer. 2018 Sep;7(3):235-60.

2 Ministry of Health of the People's Republic of China. Guidelines on the Diagnosis and Treatment of Primary Liver Cancer (2011 Edition). J Clin Hepatol. 2011;27:1141-59.

3 Chen W, Zheng R, Baade PD, Zhang S, Zeng H, Bray F, et al. Cancer statistics in China, 2015. CA Cancer J Clin. 2016 Mar-Apr;66(2):115-32.

4 El-Serag HB, Rudolph KL. Hepatocellular carcinoma: epidemiology and molecular carcinogenesis. Gastroenterology. 2007 Jun;132(7):2557-76. 